\title{
The Ubiquitous Use of Resting State as a Control Task for Language Mapping in Task-Based Functional MRI
}

$\mathbf{W}$ e read with great interest the article entitled, "LesionSpecific Language Network Alterations in Temporal Lobe Epilepsy," by Foesleitner et al. ${ }^{1}$ Functional connectivity, often synonymously used in reference to "resting-state functional connectivity," offers a unique insight into brain connections and has shown some promise in better understanding of normal and abnormal brain function, which complements information that can be ascertained from task-based fMRI studies. Indeed, the authors found distinct functional connectivity profiles in these different epilepsy cohorts that were not ascertainable by taskbased fMRI analysis, which may lead to a better understanding of how epilepsy affects language organization. However, we have concerns about the study design and assertions by the authors.

First, the use of a "rest" control block is relatively ubiquitous in clinical task-based fMRI for language mapping, despite its well-known effects on calculated "activation.", Binder et $\mathrm{al}^{2}$ have previously shown considerable overlap in activation patterns when contrasting a resting state with a tone-decision task and a semantic-decision task with the same tone control task. Critically, when one contrasts the semantic decision task with rest, expected areas of language activation are no longer observed. ${ }^{2}$ Using rest as a control condition can result in false-negative activation maps and erroneous calculation in the laterality index. Most important, the strongest validation for the laterality index compared with Wada and verbal memory outcomes is by use of such an active control task (semantic-decision versus tone-decision task).

Multiple factors likely contribute to the apparent loss of activation when using rest as the language control compared with an active control task. Because the resting state is not truly devoid of synchronized neuronal activity, it is a false assumption that rest is somewhat of a "blank canvas" with which cognitive functions can be compared. So-called task-negative networks (eg, default mode network $[\mathrm{DMN}]$ ) show greater activity during the rest state and are relatively suppressed at the onset of a cognitive task. Thus, during a block design (as performed by the authors), one can model these task-negative networks as "off" during the language task block and "on" during the rest block, resulting in its own "block design," which is anticorrelated to the task of interest. Therefore, these areas of task-negative network activation will be subtracted

http://dx.doi.org/10.3174/ajnr.A6464 from the language task activation, giving an erroneous calculation of task activation. Most important, this adds an additional source of subject-specific variability because the degree of deactivation of these networks has been shown to be dependent on the perceived difficulty of the task. ${ }^{4}$ While there is debate on whether the DMN functions in the semantic network, ${ }^{4}$ the effect of task-negative network activation is critical, nevertheless, because it shares similar anatomic overlap with areas of semantic processing; therefore, rest is generally an inappropriate control for language mapping.

Second, we also have concerns about the use of resting blocks in a task-based acquisition for functional connectivity because these are known not to be entirely representative of true dedicated resting acquisitions. ${ }^{5}$ The authors do not clearly discuss this point, making extrapolation to more widely used dedicated resting-state scans problematic. Our greater concern is the suggestion by the authors that the strategy of using resting blocks from a task acquisition is advantageous because it adds no additional scan time. The encouragement to use rest as a control block for task-based language fMRI may potentially have profound adverse effects on decision-making in epilepsy surgery with regard to risks of verbal memory decline and should be discouraged.

Last, while their results raise interesting questions about the pathophysiology of language function and organization in the setting of various epilepsy cohorts, it is critical to remember that we use laterality index with task-based fMRI to assess surgical risks. Because no such outcome data are presented in this study, one must exercise caution in assuming that these functional connectivity findings have any role in assessing risks for epilepsy surgery and cannot replace task-based fMRI until such validation has occurred.

Disclosures: Erik Middlebrooks_UNRELATED: Grants/Grants Pending: Varian Medical Systems and Boston Scientific Corp, Comments: Both entities provided research support unrelated to this work.* *Money Paid to the Institution.

\section{REFERENCES}

1. Foesleitner O, Nenning KH, Bartha-Doering L, et al. Lesion-specific language network alterations in temporal lobe epilepsy. AJNR Am J Neuroradiol 2020;41:147-54 CrossRef Medline

2. Binder JR, Swanson SJ, Hammeke TA, et al. A comparison of five fMRI protocols for mapping speech comprehension systems. Epilepsia 2008; 49:1980-97 CrossRef Medline

3. Middlebrooks EH, Yagmurlu K, Szaflarski JP, et al. A contemporary framework of language processing in the human brain in 
the context of preoperative and intraoperative language mapping. Neuroradiology 2017;59:69-87 CrossRef Medline

4. Humphreys GF, Hoffman P, Visser M, et al. Establishing task- and modality-dependent dissociations between the semantic and default mode networks. Proc Natl Acad Sci U S A 2015;112:7857-62 CrossRef Medline

5. Ganger S, Hahn A, Kublbock M, et al. Comparison of continuously acquired resting state and extracted analogues from active tasks. Hum Brain Mapp 2015;36:4053-63 CrossRef Medline
(D) E.H. Middlebrooks Departments of Radiology and Neurosurgery Mayo Clinic Jacksonville, Florida

(DD.S. Sabsevitz Department of Neuropsychology Mayo Clinic Jacksonville, Florida 